Title:

Author(s):

Submitted to:

\title{
Applied Mathematics of Chaotic Systems
}

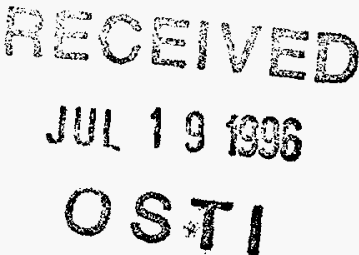

Erica Jen, T-7

Mark Alber, University of Notre Dame

Roberto Camassa, T-7

Wooyoung Choi, T-7

James Crutchfield, University of California, Berkeley

Darryl Holm, T-7

G. Kovacic, RPI

Jerrold Marsden, University of California, Berkeley

\section{DOE Office of Scientific and Technical} Information (OSTI)

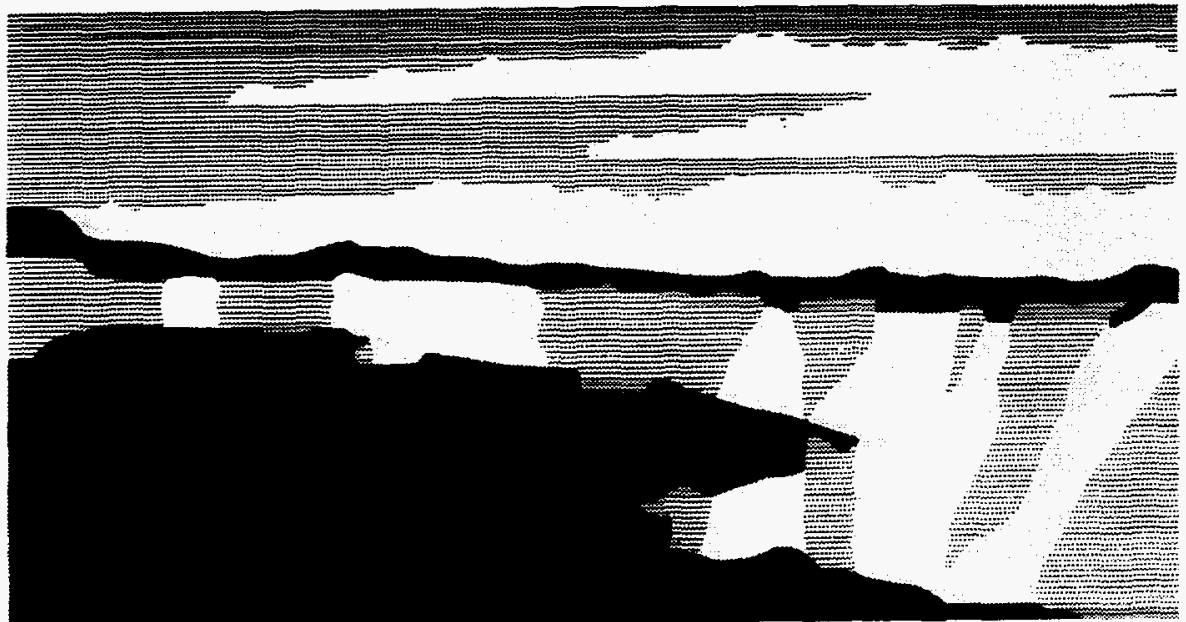

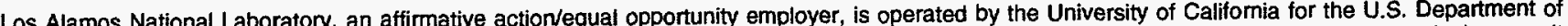
Los Alamos National Laboratory, an aftirmative actionequal opportunity employer, is recognizes that the U.S. Government retains a nonexclusive, royaltyfree license to publish or reproduce the published form of this contribution, or to allow others to do so, for U.S. Government purposes. The Los Alamos

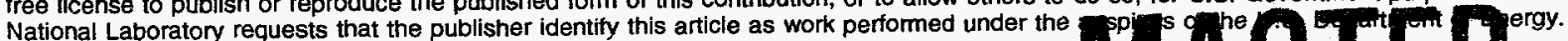




\section{DISCLAIMER}

Portions of this document may be illegible in electronic image products. Images are produced from the best available original document. 


\title{
Applied Mathematics of Chaotic Systems
}

Erica Jen*, Mark Alber (University of Notre Dame), Roberto Camassa, Wooyoung Choi, James Crutchfield (UC, Berkeley), Darryl Holm, G. Kovacic, and Jerrold Marsden (UC, Berkeley)

\begin{abstract}
This is the final report of a three-year, Laboratory-Directed Research and Development (LDRD) project at the Los Alamos National Laboratory (LANL). The objectives of the project were to develop new mathematical techniques for describing chaotic systems and for reexpressing them in forms that can be solved analytically and computationally. We focused on global bifurcation analysis of rigid body motion in an ideal incompressible fluid and on an analytical technique for the exact solution of nonlinear cellular automata. For rigid-body motion, we investigated a new completely integrable partial differential equation (PDE) representing model motion of fronts in nematic crystals and studied perturbations of the integrable PDE. For cellular automata with multiple domain structures, our work has included: 1) identification of the associated set of conserved quantitites for each type of domain; 2) use of the conserved quantitites to construct isomorphism between the nonlinear system and a linear template; and 3) use of exact solvability methods to characterize detailed structure of equilibrium states and to derive bounds for maximal transience times.
\end{abstract}

\section{Background And Research Objectives}

Within the past decade substantial progress has been made in the understanding of many physical phenomena long considered intractable. Representative examples include the onset of turbulence in fluid flows, and the behavior of unstable chemical reacting fronts such as flames. These phenomena are typified by features that defy complete analytic treatment, such as strong nonlinearities, apparent randomness of behavior, and wildly fluctuating regions of stability. The recent progress in these problems has been achieved through an amalgam of

*Principal investigator, e-mail: erica@lanl.gov 
theoretical, experimental, and computational studies, with a leading role played by significant advances in the development of applied mathematics for chaotic systems.

This project focuses on the development of new concepts and techniques of applied mathematics for describing and solving two specific systems: rigid-body motion through an incompressible fluid and one-dimensional cellular automata.

\section{A. Global Bifurcation Theory of Low-Dimensional Systems}

The motion of a rigid body immersed in an ideal fluid at rest at infinity can be described, quite remarkably, by a set of six ordinary differential equations (ODEs), named after Kirchhoff who first provided an elegant derivation. The effects of the fluid on the body enter the equations as constant terms in inertia-like tensors, physically representing the added mass the body feels in displacing the fluid as it moves. The motion of the fluid completely decouples from the motion of the body, and can in principle be reconstructed by solving the Laplace equation with appropriate boundary conditions, once a solution to the Kirchoff ODEs for the body motion is known. The problem is to investigate the parameters and conditions under which these equations lead to chaotic dynamics.

\section{B. Exact Solution of Cellular Automata}

Cellular automata (CA) are a class of dynamical systems characterized by discreteness, determinism, local interaction, and an inherently parallel form of evolution. Specifically, CA are defined as lattices of processors whose site values are determined at any time step by the values of their neighboring processors at the previous time step.

The complexity and diversity of behavior generated by CA have motivated widespread use of these systems in the modeling of complex phenomena and in the design of parallelprocessing algorithms. The applications of these discrete systems are hampered, however, by the lack of a theoretical foundation. There is an urgent need for the development of analytical techniques for the understanding of phenomena such as transient behavior, stability of orbits, and pattern formation.

\section{Importance To Lanl's Science And Technology Base And National R\&D Needs}

Although highly idealized, the problem of rigid-body motion is relevant for testing any numerical computer program devised to deal with the more realistic situation of a viscous fluid, with superimposed flows and in the presence of boundaries other than the rigid body. Such a computer program is being implemented by a group at the University of Illinois at Urbana to study the problem of convection of chunks of ice in jet engines, for the purpose of protecting 
the turbine blades during aircraft de-icing procedures. Furthermore, the technical difficulties that have to be overcome in the analysis of the corresponding system of ordinary differential equations lead to introduction of new theoretical methods in dynamical systems theory.

Cellular automata, the second component of the project, are important theoretically as prototypical, spatially-extended, discrete dynamical systems - other examples of these systems include lattice gases, coupled lattice maps, neural nets, and sandpile models. Practically, CA are useful in the modeling of the type of complex behavior generated by the interaction of many simple, local connected components-such as multiphase flows and percolation through porous media-and in the design of parallel-processing algorithms for problems such as pattern recognition. The study of exact solutions of CA is therefore expected to contribute significantly to our understanding of the uses of these systems in modelling a wide range of complex phenomena.

\section{Scientific Approach and Results}

\section{A. Global Bifurcation Theory of Low-Dimensional Systems}

We have analyzed the hyperbolic structures for the dynamics of a class of triaxial ellipsoidal bodies that we call the Kirchhoff and C. Neumann tops [1]. Using the technique of multi-dimensional Melnikov function and global bifurcation analysis, we have identified the relevant bifurcation parameters and sets of initial conditions leading to chaotic dynamics, which has been (qualitatively) described. There are two additional main results that have come out of this study. First, by examining the nature of the transverse intersections of stable and unstable manifolds from a global point of view, we have shown that, remarkably, not all of the intersections are responsible for non-integrability by generating chaotic motion. Second, this global point of view has provided an explicit example (and closed form solutions) for geometric singular perturbation theory [1].

During the last decade several surprising connections have been found between the $\mathrm{C}$. Neumann problem, the geodesic motion on quadrics, and the stationary flows of completely integrable partial differential equations (PDEs). In recent work we have found an unexpected new connection that produces a new completely integrable PDE [2]. The quasiperiodic solutions of this equation can be viewed as geodesic motion on a quadric surface [3]. The new equation belongs to the hierarchy of the Dym equation, and physically it can be derived as a simple model for the motion of fronts in nematic crystals. For the case when the quadric surface is an ellipsoid, geodesic orbits can be mapped into orbits of the C. Neumann top. We are studying whether we can obtain new solutions of the PDEs in this class using the orbits we 
have examined for the C. Neumann case and, in particular, ascertaining whether the perturbed C. Neumann orbits correspond to solutions of (perturbed versions of) the integrable PDE.

The need to analyze orbits in the neighborhood of a saddle center fixed point in the $\mathrm{C}$. Neumann problem has also led to the introduction of a new technique for finding homoclinic orbits, which experience many excursions before settling down to a fixed point or periodic orbit [4].

\section{B. Exact Solution of Cellular Automata}

Under evolution of certain nonlinear automata, typically termed "chaotic," it has been found that the lattice of processors typically "organizes" itself into multiple contiguous domains within which behavior is ordered and highly correlated. The domains are separated by domain walls that may be interpreted physically as dislocations or, equivalently, as propagators of information.

The analysis of domain structure and dislocation behavior has led to the development of a solution method [5] for a wide class of nonlinear automata. The method maps the nonlinear data onto an exactly solvable linear "template" automaton with a closely-related evolution function.

A critical component of the mapping between nonlinear and linear systems is the characterization of the behavior of the dislocation processors. For the prototypical cellular automaton Rule 18 studied in the first stage of this project, it has been shown $[5,6]$ that the dislocations are analogous to classical, annihilating diffusive particles. The propagation of these particles provide a realization of quasi-periodicity in these discrete dynamical systems $[5]$.

Using the solution method developed for cellular automaton Rule 18, we have obtained results [7] for the expected number of dislocations in an arbitrary finite lattice; the expected transience time for collision and annihilation of dislocations; and maximal bounds on transience time for this cellular automaton. The results represent one of the very few analytical characterizations of nonequilibrium behavior for nonlinear, spatially-extended dynamical systems.

In addition, a technique has been developed to characterize the "grammar" that generates the set of spatial sequences that appear in domain structures stable under evolution of Rule 54. It has moreover been shown that all sequences recognized by this grammar are attracted to limit cycles of identical period. A corollary of the result provides a proof of the fact that the rule acts upon a certain well-defined subset of spatial sequences so as to induce a spatial reflection of those sequences. The result represents the first demonstration of a global reflection operator induced by a local interaction rule. 
The focus of the project in its final year has been on completing the development and extension of the exact solvability method. In particular, for Rule 54 (for which multiple domain structures have been found) and for Rule 22 (for which preliminary computational evidence indicates a similar and possibly related taxonomy of domain structures), the goal has been to 1) identify for each type of domain structure the associated set of conserved quantities, 2) use the exact solvability method to derive exact expressions for limit cycle periods for attractors of the rules, and to derive bounds for maximal transient times.

\section{References}

[1.] R. Camassa and D. Holm, "The Hyperbolic Structure of the Clebsch Top," in preparation.

[2.] M. Alber, R. Camassa, D. Holm and J. Marsden, "The Geometry of Peaked Solitons and Billiard Solutions of a Class of Integrable PDEs," Lett. Math. Phys., 32, 137 (1994).

[3.] M. Alber, R. Camassa, D. Holm and J. Marsden, "On the Link Between Umbilic Geodesics and Soliton Solutions of Nonlinear PDEs," Proc. R. Soc. Lon., Ser. A 450, 677 (1995).

[4.] R. Camassa, G. Kovacic, and S. K. Tin, "A Melnikov Method for Homoclinic Orbits with Many Pulses," Arch. Rat. Mech. Anal., submitted for publication (1995).

[5.] Erica Jen, "Aperiodicity in One-Dimensional Cellular Automata," Physica D, 45, 3 (1991).

[6.] Erica Jen, "Exact Solvability and Quasiperiodicity in One-Dimensional Cellular Automata," Nonlinearity, 4, 251 (1992).

[7.] Erica Jen, "Transience and Dislocations in One-Dimensional Cellular Automata," Les Houches Physics Advances (1993).

[8.] Erica Jen and James P. Crutchfield, "Particles and Backgrounds in Cellular Automata," Comm. Math. Phys., submitted for publication (1995).

\section{DISCLAIMER}

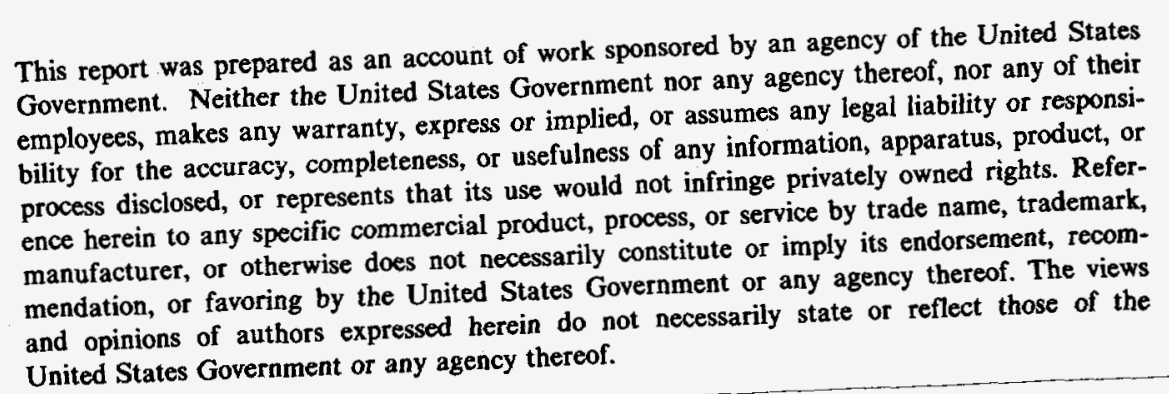

